\title{
Cochrane
}

Library

Cochrane Database of Systematic Reviews

\section{Factors influencing the implementation of early discharge hospital at home and admission avoidance hospital at home: a qualitative evidence synthesis (Protocol)}

Gearon E, O'Connor D, Wallis J, Han JX, Shepperd S, Mäkelä P, Buchbinder R

Gearon E, O'Connor D, Wallis J, Han JX, Shepperd S, Mäkelä P, Buchbinder R.

Factors influencing the implementation of early discharge hospital at home and admission avoidance hospital at home: a qualitative evidence synthesis (Protocol).

Cochrane Database of Systematic Reviews 2021, Issue 3. Art. No.: CD014765.

DOI: 10.1002/14651858.CD014765.

www.cochranelibrary.com

Factors influencing the implementation of early discharge hospital at home and admission avoidance hospital at home: a qualitative evidence synthesis (Protocol)

Copyright @ 2021 The Cochrane Collaboration. Published by John Wiley \& Sons, Ltd. 
TABLE OF CONTENTS

HEADER 1

ABSTRACT

BACKGROUND

Figure 1.

OBJECTIVES

METHODS

ACKNOWLEDGEMENTS

REFERENCES

APPENDICES

HISTORY

CONTRIBUTIONS OF AUTHORS

DECLARATIONS OF INTEREST

SOURCES OF SUPPORT 
[Qualitative Protocol]

\section{Factors influencing the implementation of early discharge hospital at home and admission avoidance hospital at home: a qualitative evidence synthesis}

Emma Gearon 1,2, Denise O'Connor1,2, Jason Wallis 1,2 , Jia Xi Han¹,2, Sasha Shepperd³, Petra Mäkelä4, Rachelle Buchbinder 1,2

1Department of Epidemiology and Preventive Medicine, School of Public Health and Preventive Medicine, Monash University, Melbourne, Australia. ${ }^{2}$ Monash Department of Clinical Epidemiology, Cabrini Institute, Malvern, Australia. ${ }^{3}$ Nuffield Department of Population Health, University of Oxford, Oxford, UK. ${ }^{4}$ Department of Health Services Research and Policy, London School of Hygiene \& Tropical Medicine, London, UK

Contact address: Jason Wallis, jwallis@cabrini.com.au, jason.wallis@monash.edu.

Editorial group: Cochrane Effective Practice and Organisation of Care Group. Publication status and date: New, published in Issue 3, 2021.

Citation: Gearon E, O'Connor D, Wallis J, Han JX, Shepperd S, Mäkelä P, Buchbinder R. Factors influencing the implementation of early discharge hospital at home and admission avoidance hospital at home: a qualitative evidence synthesis (Protocol). Cochrane Database of Systematic Reviews 2021, Issue 3. Art. No.: CD014765. DOI: 10.1002/14651858.CD014765.

Copyright $@ 2021$ The Cochrane Collaboration. Published by John Wiley \& Sons, Ltd.

\section{A B S T R A C T}

\section{Objectives}

This is a protocol for a Cochrane Review (qualitative). The objectives are as follows:

1) To identify, appraise and synthesise qualitative research evidence on the factors that influence the implementation of i) admission avoidance hospital at home and ii) early discharge hospital at home, from the perspective of multiple stakeholders, including policy makers, health service managers, health professionals, patients, and patients' caregivers.

2) To explore how our synthesis findings relate to, and help to explain, the findings of the Cochrane intervention reviews of admission avoidance hospital at home and early discharge hospital at home services. 


\section{B A C K G R O U N D}

\section{Description of the topic}

Two related 'hospital at home' services provide alternatives to traditional in-hospital care: admission avoidance hospital at home and early discharge hospital at home. Admission avoidance hospital at home avoids hospitalisation by providing acute or subacute treatment in a patient's home or usual place of residence for a limited time, for a condition that would otherwise require a hospital admission (Shepperd 2016). Eligible patients are typically referred from an emergency department, an acute assessment unit, or directly from ambulance services; they can also be referred by community physicians and specialists to receive active treatment from healthcare professionals in their homes. Early discharge hospital at home involves supporting patients to go home earlier than usual to receive acute care or sub-acute care in their homes for a limited time period (Caplan 2012; Goncalves-Bradley 2017). Eligible patients are typically referred from acute inpatient care and provided active treatment from healthcare professionals in their homes, and therefore spend less time in hospital.

A Cochrane Review of admission avoidance hospital at home included 16 randomized trials and 1814 participants with various conditions (e.g. older adults requiring admission to hospital following a stroke, with a diagnosis of dementia; or adults with chronic obstructive pulmonary disease or heart failure) (Shepperd 2016). Compared to inpatient care, the review found admission avoidance hospital at home probably makes little or no difference in risk of death or likelihood of hospital readmission (moderatecertainty evidence); may reduce admission to long-term residential aged care at six months' follow-up (low-certainty evidence); may improve patient satisfaction (low-certainty evidence); and may reduce healthcare costs (low-certainty evidence) (Shepperd 2016).

For early discharge hospital at home, findings reported in a Cochrane Review varied by health condition (Goncalves-Bradley 2017). A total of 32 randomized trials were included, with over 4700 patients with various conditions (e.g. stroke survivors, patients following elective surgery, older patients with various medical conditions such as chronic obstructive pulmonary disease). Compared to inpatient care, the review found early discharge hospital at home probably reduces length of stay for people recovering from a stroke (about 7 days), people following elective surgery (about 4 days), and older people with a medical condition ranging from half a day to 20 days (moderate-certainty evidence) . The evidence also showed these services probably make little or no difference to risk of death for people recovering from a stroke or older people with a mix of medical conditions (moderate-certainty evidence), may make little or no difference to risk of hospital readmission for people recovering from a stroke or elective surgery (low-certainty evidence), and may decrease the risk of living in long-term residential care for people recovering from a stroke and older people with a mix of medical conditions (low-certainty evidence). The review also found that early discharge hospital at home may make little or no difference to caregiver burden for people recovering from stroke or elective orthopedic surgery (lowcertainty evidence), may slightly improve patient satisfaction for people recovering from stroke or elective surgery (low-certainty evidence), and we do not know if these services reduce healthcare costs across the various conditions because the certainty of this evidence is very low (Goncalves-Bradley 2017).

Outcomes from these two Cochrane intervention reviews suggest that hospital at home services may provide either superior or similar outcomes compared to inpatient care. However, these reviews did not address how to implement these services. While health systems around the world vary with respect to financing (e.g. multiple- or single-payer systems), policy objectives for admission avoidance hospital at home and early discharge hospital at home services are likely to be similar as they are expected to reduce demand for inpatient hospital beds, reduce costs and conserve health outcomes. This review seeks to understand the factors influencing their implementation. Here we use the term implementation to describe both the process by which these models of care are introduced to health systems and hospitals by policy makers, healthcare providers and governments through policy, guidelines and financing; and also the process by which these models of care are introduced and integrated into clinical practice by clinical leads and health professionals, how patient groups are selected for the services and how the services are experienced by patients and their caregivers.

\section{How the intervention might work}

To integrate potential findings of this qualitative evidence synthesis with the two Cochrane intervention reviews we have developed a draft logic model (Baxter 2014). The purpose of a logic model is to outline the hypothesised causal pathway that links the intervention (e.g. admission avoidance hospital at home for eligible patients) with mediating factors that impact on the key outcomes (e.g. health status, healthcare utilisation, patient and caregiver satisfaction, admission to long-term residential care, as reported in the Cochrane intervention reviews) (Figure 1). 
Figure 1. Logic model describing the factors that influence implementation of admission avoidance hospital at home and early discharge hospital at home

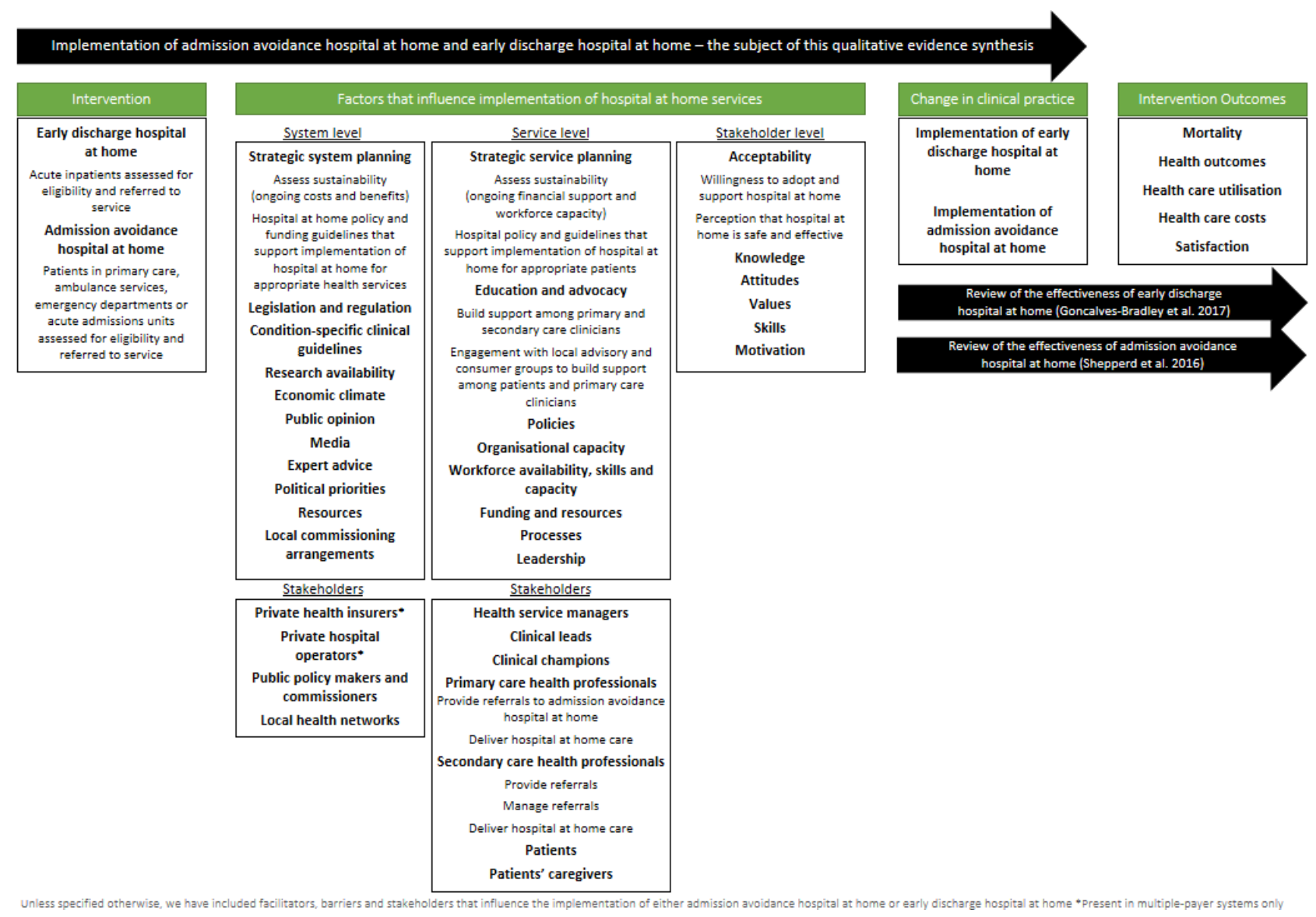

As a preliminary step we have integrated the findings of qualitative studies known to the review team into the draft logic model to suggest potential mediating factors and key stakeholders at the system, service, and stakeholder level that may influence implementation of hospital at home services (Gardner 2003; Lemelin 2007; Kraut 2016; Buhagiar 2017; Mäkelä 2018; Brody 2019; Dismore 2019; Gardner 2019). This logic model suggests that the key stakeholders for successful implementation vary in multiple-payer systems and single-payer systems at the system level (e.g. private health insurers and public policy makers) but hold similar positions at the service level (e.g. health service managers and health professionals in primary and secondary care). We expect this logic model to evolve alongside the qualitative evidence synthesis, and we will invite members of our stakeholder advisory panel to review the findings. This will ensure we include interpretations from a broader lens in development of the logic model.

\section{Why is it important to do this synthesis?}

There is substantial variation in the implementation of hospital at home services across different countries and healthcare settings. Across England, a single-payer system, healthcare providers have implemented three different type of hospital at home services, each with varying functions. This includes, admission avoidance hospital at home, early discharge hospital at home, and discharge to assess (services that support patients to be discharged to their own home or a community setting) (Young 2009). This variation makes it difficult to assess how hospital at home services contribute to healthcare and ease the demand for hospital-based care (NHS Benchmarking Network 2015). In Australian public hospitals (a single-payer system), hospital in the home multi-day admissions ranged from 25 admissions in Tasmania in 2017 to 2018 to 30,070 admissions in Victoria in the same year (Australian Institute of Health and Welfare 2019). Given the observed variation in implementation of admission avoidance hospital at home and early discharge hospital at home, and possibly variation in thresholds for admission to hospital at home, there is a need to understand the factors that influence implementation of these models and how these may differ between healthcare settings (for example, highincome versus low- and middle-income countries; single-payer systems versus multiple-payer systems; urban versus regional or rural contexts; mechanisms of referral and boundaries with other health services; and the provision of social care and admission criteria). Where data permit, these factors will be explored in the qualitative evidence synthesis from the perspectives of multiple stakeholders.

To the best of our knowledge, while there have been individual qualitative studies investigating the barriers and facilitators that influence implementation of admission avoidance hospital at home and early discharge hospital at home services (Gardner 2003; Lemelin 2007; Kraut 2016; Buhagiar 2017; Mäkelä 2018; Brody 2019; Dismore 2019; Gardner 2019), there has been no overarching 
synthesis of qualitative studies exploring these factors. We propose such a review that will integrate and enhance the findings of the two Cochrane intervention reviews.

\section{How this review might inform or supplement what is already known in this area}

A qualitative exploration using a logic model to guide analysis will facilitate interpretation of the findings from the Cochrane intervention reviews by identifying the factors that hinder or support the implementation of these services. An assessment of the factors that influence the implementation of these services from the perspectives of people involved in the funding, commissioning and delivery of care (e.g. policy makers, managers, health professionals), and people receiving care (e.g. patients and caregivers), may also help to explain the reasons for variation in implementation of admission avoidance hospital at home and early discharge hospital at home in different health settings.

This qualitative evidence synthesis will take a global perspective; the review of the evidence will not be limited to one country. The findings will assist implementation of admission avoidance hospital at home and early discharge hospital at home, and will be beneficial for a range of stakeholders, including public policy makers and commissioners, health insurers, health service managers, health professionals, patients, and patients' caregivers.

\section{O B JECTIVES}

1) To identify, appraise and synthesise qualitative research evidence on the factors that influence the implementation of i) admission avoidance hospital at home and ii) early discharge hospital at home, from the perspective of multiple stakeholders, including policy makers, health service managers, health professionals, patients, and patients' caregivers.

2) To explore how our synthesis findings relate to, and help to explain, the findings of the Cochrane intervention reviews of admission avoidance hospital at home and early discharge hospital at home services.

\section{METHODS}

\section{Design}

A qualitative evidence synthesis of primary qualitative studies. Study reporting will be guided by the Cochrane Effective Practice and Organisation of Care (EPOC) qualitative evidence synthesis template (Glenton 2020) and the 'enhancing transparency in reporting the synthesis of qualitative research' (ENTREQ) guidance (Tong 2012).

\section{Criteria for considering studies for this review}

\section{Types of studies}

We will include primary studies that used qualitative methods for both data collection (such as focus group discussions or individual interviews) and data analysis (such as thematic analysis, framework analysis and grounded theory). We will exclude studies that collected data using qualitative methods but did not analyse these data using qualitative analysis methods. As we expect to find sufficient qualitative studies that use qualitative methods for both data collection and data analysis, we will exclude studies that collected data using open-ended survey questions.
We will include both published and unpublished studies and studies published in any language. Mixed-method studies will be included where it is possible to extract the data that were collected and analysed using qualitative methods. We will not exclude studies based on our assessment of methodological limitations. We will use this information about methodological limitations to assess our confidence in the review findings. We will include studies regardless of whether they were conducted alongside studies of the effectiveness of admission avoidance hospital at home and early discharge hospital at home or not (Shepperd 2016; GoncalvesBradley 2017).

\section{Topic of interest}

The review will include qualitative studies that examine the implementation of new or existing hospital at home services (admission avoidance hospital at home or early discharge hospital at home) from the perspective of different stakeholders. This will allow exploration of factors influencing implementation of hospital at home services that are being introduced compared with services that are already in place. We use the term 'implementation' to describe both the process by which these models of care are introduced to health systems and hospitals through policies and guidelines, and also the process by which these models of care are put into clinical practice by clinical leads and teams of health professionals for appropriate patients and accepted by patients and patients' caregivers.

To ensure the focus of this qualitative evidence synthesis matches the focus of the corresponding Cochrane intervention reviews, we have used similar definitions and exclusion criteria (Shepperd 2016; Goncalves-Bradley 2017).

\section{Types of interventions}

Admission avoidance hospital at home is a service that is established to avoid hospitalisation. It provides acute or subacute treatment in a patient's home for a limited time, for a condition that would otherwise require a hospital admission (Shepperd 2016). We will include studies where patients are admitted to a hospital at home service from primary care in the community, ambulance services or from hospital emergency departments or acute admissions units.

Early discharge hospital at home is a service that provides health care for patients discharged early from hospital. It provides acute or subacute treatment in a patient's home for a limited time, for a condition that would otherwise require a hospital admission (Deloitte Access Economics 2011; Goncalves-Bradley 2017). We will include studies where patients are admitted to this service following an early discharge from hospital. We will consider early discharge as defined by the authors of included studies as there is no consistent definition; the length of hospital admission prior to early discharge varies by health condition and varies across trials (Deloitte Access Economics 2011).

We will include studies about admission avoidance hospital at home and early discharge hospital at home where health professionals provide active acute or subacute treatment in a patient's home or usual place of residence for a limited time and for a condition that would otherwise require a hospital admission. Typically, patients remain under the clinical responsibility of a hospital clinician while receiving hospital at home treatment, but we will also include studies where a patient's general practitioner

Factors influencing the implementation of early discharge hospital at home and admission avoidance hospital at home: a qualitative 
takes clinical responsibility. Health professionals that deliver hospital at home care can be hospital employees or employed through a service in the community (e.g. district nurses). For the purpose of this review, we define acute care as active, urgent, short-term treatment in which the principal intent is to relieve symptoms of illness or injury, reduce the severity of an illness or injury, or protect against exacerbation or complication of an illness or injury that could threaten life or normal function (OECD 2011, Independent Hospital Pricing Authority 2015). We define subacute care as multidisciplinary care that is delivered by or informed by health professionals with specialist expertise and includes negotiated goals within a specified time frame that aim to optimise the patient's functioning and quality of life (OECD 2011; Independent Hospital Pricing Authority 2015).

In line with these definitions, and with the exclusion criteria of the corresponding Cochrane intervention reviews, this review will exclude studies that include patients not deemed to require acute or subacute care in a hospital setting. For example, we will exclude from this review services that provide long-term patient care, care in outpatient settings or after discharge from hospital, palliative care, and self-care by the patient in their home (Shepperd 2016; Goncalves-Bradley 2017). We will additionally exclude studies that focus only on obstetric care, paediatric care, palliative care at home, or mental health hospital at home (such as crisis-resolution hospital at home) to ensure the focus of this qualitative evidence synthesis matches the focus of the corresponding Cochrane intervention reviews (Shepperd 2016; Goncalves-Bradley 2017). Studies that explore implementation of hospital at home services for both adults (aged 18 years or older) and children will be included where data relating to adult hospital at home services are reported separately.

\section{Types of participants}

The review will include the perspective of multiple stakeholders involved in admission avoidance hospital at home and early discharge hospital at home. Stakeholders' perspectives encompass their experiences, beliefs, perceptions and views relating to the implementation of admission avoidance hospital at home and early discharge hospital at home services. At the system level these stakeholders can include the following.

- Policy makers and commissioners (or people in similar roles who make decisions about funding and policies for admission avoidance hospital at home and early discharge hospital at home)

- Private health insurers (or people in similar roles in multiplepayer systems who make decisions about funding and policies for admission avoidance hospital at home and early discharge hospital at home)

- Private hospital operators (or people in multiple-payer hospitals and hospital networks who make decisions about hospital policy and funding for admission avoidance hospital at home and early discharge hospital at home)

- Local health networks (or people in single-payer hospitals and hospital networks) who make decisions about hospital policy and funding for admission avoidance hospital at home and early discharge hospital at home

At the service level these stakeholders can include the following.
- Health service managers (people in single-payer or multiplepayer hospitals who make decisions about policy and funding for patients' care)

- Clinical leads (health professionals who co-ordinate and supervise teams of health professionals)

- Clinical champions (health professionals who advocate for admission avoidance hospital at home and early discharge hospital at home to help with successful implementation)

- Primary care health professionals (health professionals in the community that provide referrals to admission avoidance hospital at home; health professionals in the community who deliver hospital at home care)

- Secondary care health professionals (health professionals in the hospital emergency department that provide referrals to admission avoidance hospital at home; health professionals in the hospital that provide referrals to early discharge hospital at home; health professionals in the hospital that manage referrals to admission avoidance hospital at home and early discharge hospital at home; health professionals in the hospital that deliver hospital at home care)

- Adult patients (aged 18 years and older) with a disease or condition requiring an acute or subacute hospital admission, who are eligible for admission avoidance hospital at home or early discharge hospital at home. Patients requiring these services for the sole purpose of palliative care, obstetric care, or mental health hospital at home are excluded from this review (see types of interventions)

- Patients' caregivers (adults who would primarily provide assistance with activities of daily living, personal care and navigating services for the patient during the period of recovery) (Talley 2007; Mäkelä 2020).

\section{Search methods for identification of studies}

The EPOC Information Specialist will develop the search strategy for this review in consultation with the review authors (Noyes 2020).

\section{Electronic searches}

To identify primary studies we will search in MEDLINE, Ovid; CINAHL, EbscoHOST; Scopus, Elsevier; and the Global Index Medicus, WHO. We will develop search strategies for each database, and will not apply limits to language or publication date. Our search strategy will combine known terms for admission avoidance hospital at home and early discharge hospital at home with a qualitative filter. Terms related to admission avoidance hospital at home and early discharge hospital at home will be derived from a "gold set" of known qualitative papers that will be included in the qualitative evidence synthesis, as well as all terms used in included papers in the Cochrane intervention reviews (Shepperd 2016; Goncalves-Bradley 2017), and terms currently used in real-world practice and evaluations (HITH Society Australasia 2019; World Hospital At Home Congress 2019). Our strategy favours specificity over sensitivity (Harris 2018). The proposed MEDLINE strategy is detailed in Appendix 1, which we will adapt for other databases. We will provide appendices for all strategies used.

\section{Searching other resources}

Our search will be supplemented by searching the reference list of relevant articles, and forward tracking the citations of studies included in the qualitative evidence synthesis and key references (the Cochrane intervention reviews and their included trials) using

Factors influencing the implementation of early discharge hospital at home and admission avoidance hospital at home: a qualitative 
Scopus. Additionally, we will approach experts in the field to request studies that might meet our inclusion criteria.

\section{Grey literature}

We will conduct a grey literature search of the following resources to identify studies not indexed in the databases listed above.

- OpenGrey (www.opengrey.eu)

- Grey Literature Report (New York Academy of Medicine; www.greylit.org)

- BASE (www.base-search.net)

\section{Selection of studies}

Two review authors will independently screen the title and abstract of all potentially eligible studies, and code them as 'retrieve' (potentially eligible or unclear) or 'do not retrieve' (not eligible). Two review authors will independently screen the full text of all retrieved articles and identify studies for inclusion and record reasons for exclusion of ineligible studies. Any disagreement will be resolved through discussion, and if need be a third author will be available to adjudicate. Where necessary, study authors will be contacted for further information. We will include a table listing studies that we excluded from our qualitative evidence synthesis at the full-text stage, along with the main reasons for exclusion. Where the same study (i.e. using the same sample and methods) has been presented in different reports, we will collate these reports so that each study, rather than each report, is the unit of interest in our qualitative evidence synthesis. Covidence will be used to screen and select studies, and we will include a Preferred Reporting Items of Systematic Reviews and Meta-analysis (PRISMA) flow diagram (Liberati 2009).

\section{Language translation}

We will include titles and abstracts of papers in any language. For titles and abstracts that are published in a language other than English, we will use open source software (google translate) to determine whether the paper should be included. We will retrieve the full text of papers that appear to fit the inclusion criteria or for which we are uncertain. We will ask members of Cochrane networks or other networks that are fluent in that language to assist in assessing the full text of the paper for inclusion. If this is not successful, the paper will be listed under 'Studies awaiting classification' to ensure transparency in the review process.

\section{Sampling of studies}

Qualitative evidence syntheses aim for variation in concepts rather than an exhaustive sample, and large amounts of study data can impair the quality of the analysis. Once we have identified all studies that are eligible for inclusion, we will assess whether their number or data richness is likely to represent a problem for the analysis and will consider selecting a sample of studies.

If sampling is required we will use purposeful sampling, which aims to limit data redundancy while ensuring optimal data richness and diversity. We will stratify studies by intervention type (admission avoidance hospital at home or early discharge hospital at home). For each intervention, we will then use a sampling frame to ensure representation by stakeholder group level (system level, local level), and geographic area (urban and regional or rural; lowand middle-income countries and high-income countries). Where we observe potential for data redundancy (for example, multiple studies of patients in urban, high-income country settings), we will assess the data richness of each study using a data richness scale (Appendix 2) (Ames 2017). Only studies that provide a good amount and depth of qualitative data pertaining to factors that influence implementation (data richness score greater than three) will be included (Cochrane EPOC 2017).

\section{Data extraction}

Two review authors will independently extract data on study characteristics, using a standardised data collection form developed for this qualitative evidence synthesis. Any discrepancies will be resolved by discussion or adjudication by a third author. For each study we will record details, where available, on the following.

- Intervention addressed (admission avoidance hospital at home; early discharge hospital at home) and whether the study is linked to an intervention trial

- Time since the intervention was implemented

- Study details (first author; corresponding author; year of evaluation; year of publication)

- Research question and aims

- Geographic setting (country of programme; low- and middleincome or high-income country classification; urban, regional or rural location; multiple-payer or single-payer system; number of hospitals)

- Participants (number; patient and caregiver characteristics (e.g. age, sex, clinical conditions), stakeholder's role or position (e.g. system level; private health insurer), service level (e.g. secondary care health professional)) and method of sampling participants

- Method of data collection (e.g. interview, focus group)

- Method of data analysis (e.g. thematic analysis)

- All text from the results sections of the included publications will be extracted verbatim, including themes, sub-themes, supporting quotes and conclusions (Thomas 2008).

To provide context for the qualitative findings we will use the Template for Intervention Description and Replication (TIDieR) framework to extract information on the admission avoidance hospital at home or early discharge hospital at home intervention that the qualitative study is embedded in (Hoffmann 2014). Where required we will extract this information from the qualitative study or from a linked effectiveness trial.

- What (materials): were any informational materials used?

- What (procedures): is there a programme manager and if so what is their role? What are the patient eligibility criteria? Who refers patients to the programme and what is the admission pathway? On average, how many days do patients spend in acute care before being transferred to hospital at home? Is acute or subacute care provided to patients in the hospital at home service?

- Who provided: who are patients under the care of while enrolled in hospital at home? Who delivers hospital at home care, and are they employed through the hospital or are they from the community? Was training provided to the health professionals that refer to hospital at home services or deliver hospital at home services? 
- How and where was the hospital at home intervention delivered (e.g. 1:1 face-to-face in patients' homes)?

- When and how much: on average, how many days do patients spend in hospital at home, and how often they received treatment by a hospital at home health professional? How long has the programme been running?

- Tailoring: was the intervention tailored to specific hospitals, health professionals or patients or patient groups, and if so how?

- Modifications: was the intervention modified during the study?

- How well (planned and actual): did the intervention employ any strategies to improve fidelity, and was fidelity achieved?

\section{Assessing the methodological limitations of included studies}

Two review authors will independently assess the methodological limitations of each included study using the Critical Appraisal Skills Programme (CASP) checklist (Critical Appraisal Skills Programme 2020). Any discrepancies will be resolved by discussion or adjudication by a third author. The CASP tool uses a checklist of 10 questions, each of which includes multiple signalling questions to help users interpret the items (29 signalling questions in total). We will summarise the findings of the CASP checklist in a 'Methodological limitations' table.

\section{Data analysis and synthesis}

We will use thematic synthesis to code the results text of included studies for factors that influence the implementation of admission avoidance hospital at home or early discharge hospital at home according to stakeholder perspectives. For example: system level (private health insurer); service level (patient, health professional, health service manager). We will use the approach recommended by Thomas and Harden including inductive line-by-line coding of extracted text and development of descriptive themes (Thomas 2008).

We will review each line of extracted text and will develop codes based on the content and meaning of each extract. Existing codes will be reviewed and revised as new codes are added. When all studies have been coded, all text related to each code will be reviewed for consistency of coding across studies. Two review authors will independently code an initial subset of studies and then meet to discuss any discrepancies until consensus is reached. The remaining studies will then be coded by one author and verified by a second author.

We will review all codes for similarities and differences, and organise them into related descriptive themes relating to the factors that influence the implementation of admission avoidance hospital at home and early discharge hospital at home services. One author will draft a summary of the descriptive themes and these will be discussed by the review team until consensus is reached. We will manage the analysis using NVivo 12 software (NVivo).

To maximise the likelihood that our findings are transferable to realworld practice (Marshall 2014), we will invite our grant stakeholder advisory panel to review the qualitative evidence synthesis findings and revised logic model (NHMRC 2017). Articulating our findings through a logic model will facilitate communication of the findings to stakeholders and identification of strategies to improve the implementation of admission avoidance hospital at home and early discharge hospital at home services (Harris 2018).

Once we have finished preparing the qualitative evidence synthesis findings, we will examine each finding, and develop prompts for future implementers. These prompts will be presented in the 'Implications for practice' section of the full review. These prompts are not intended to be recommendations but will be phrased as questions to help implementers consider the implications of the review findings within their context (i.e. to what extent are identified factors that influence the implementation of hospital at home services addressed). We will send this section and our logic model to our stakeholder advisory panel with representation from different healthcare sectors and countries, where possible, to gather their feedback about the relevance of these prompts and the manner in which they are phrased and presented.

\section{Subgroup analyses}

Where data permit, we will examine similarities and differences in the factors that influence the implementation of hospital at home services with regard to the following study characteristics.

- Type of delivery model (admission avoidance hospital at home versus early discharge hospital at home)

- Geographic setting (high-income versus low- and middleincome countries; single-payer systems versus multiple-payer systems; urban versus regional or rural contexts)

- Presence of intervention components (mechanisms of referral; the provision of social care; admission criteria)

- Patient populations where there is evidence that hospital at home services are effective (e.g. older adults requiring hospital admission, such as for chronic obstructive pulmonary disease; people recovering from stroke; people recovering from surgery), as informed by the Cochrane intervention reviews; patient populations identified as a priority for hospital at home services by policy makers.

\section{Assessment of confidence in review findings}

Two review authors will use the GRADE-CERQual (Confidence in the Evidence from Reviews of Qualitative research) approach to assess our confidence in each finding (Lewin 2018). CERQual assesses confidence in the evidence, based on the following four key components.

- Methodological limitations of included studies: the extent to which there are concerns about the design or conduct of the primary study that contributed evidence to an individual review finding

- Coherence of the review finding: an assessment of how clear and cogent the fit is between the data from the primary studies and a review finding that synthesises those data (by 'cogent', we mean well supported or compelling)

- Adequacy of the data contributing to a review finding: an overall determination of the degree of richness and quality of data supporting a review finding

- Relevance of the included studies to the review question: the extent to which the body of evidence from the primary studies supporting a review finding is applicable to the context 
(perspective or population, phenomenon of interest, setting) specified in the review question

After assessing each of these four components, we will make a judgement about the overall confidence in the evidence supporting the review finding. We will judge confidence as high, moderate, low, or very low. The final assessment will be based on consensus among the review authors. All findings start as 'high confidence', and will be graded down if there are important concerns regarding any of the CERQual components.

\section{'Summary of qualitative findings' table and evidence profile}

We will present summaries of the findings and our assessments of confidence in these findings in the 'Summary of qualitative findings' table(s). We will present detailed descriptions of our confidence assessment in evidence profile(s).

\section{Integrating the review findings with the Cochrane intervention reviews}

We will continue to develop the logic model (Figure 1) in order to integrate findings from our qualitative evidence synthesis with findings from the Cochrane intervention reviews. The findings of the qualitative evidence synthesis may inform subgroup analyses for future updates of the Cochrane intervention reviews, as well as the design of future trials aiming to implement admission avoidance hospital at home and early discharge hospital at home services.

\section{Review author reflexivity}

All review team members have prior experience with hospital at home, or an awareness that hospital at home services have either superior or similar effects compared to inpatient care but are potentially under-utilised. Hence, all team members tend to view these services favourably. We will approach the review with an awareness of this bias toward positively viewing hospital at home services, and will endeavour to keep an open and curious mind about the factors that influence the implementation of early discharge hospital at home and admission avoidance hospital at home.

We will maintain a reflexive stance throughout all stages of this review process. All decisions or processes will be conducted independently by at least two team members, who will then discuss with each other and with the review team how their own backgrounds and positions may affect the analysis and interpretation of review findings. This will allow for regular opportunities to critically examine all decisions made and to counter our own biases. Members of this review team have clinical backgrounds (JW, DOC, RB, PM), qualitative research backgrounds (DOC, JW, SS, PM, RB), experience in writing Cochrane Reviews (DOC, JW, JH, SS, RB) and experience in conducting research examining hospital in the home (EG, JW, DOC, SS, PM, RB). Through our collective and individual experiences as clinicians, academics, researchers and policy makers, we anticipate that this review will reveal a combination of organisational, professional and individual factors that influence the implementation of admission avoidance hospital at home and early discharge hospital at home. As a team, we will remain mindful of our presuppositions and will support each other to minimise the influence of these on our analysis or the interpretation of our findings. The lead author will keep a reflexive journal throughout the review process and will document and reflect on progress and decisions made (Nowell 2017).

\section{ACKNOWLEDGEMENTS}

This research is conducted by the authors for the National Health and Medical Research Council (NHMRC) Partnership Centre for Health System Sustainability (grant ID number: 9100002), administered by the Australian Institute of Health Innovation, Macquarie University. Along with the NHMRC, the funding partners in this research collaboration are: The Bupa Health Foundation; NSW Ministry of Health; Department of Health, WA; and The University of Notre Dame Australia. Their generous support is gratefully acknowledged. While the NHMRC, The Bupa Health Foundation, NSW Ministry of Health, Department of Health, WA and The University of Notre Dame Australia have provided in-kind and financial support for this research, they have not reviewed the content and are not responsible for any injury, loss or damage however arising from the use of, or reliance on, the information provided herein. The published material is solely the responsibility of the authors and does not reflect the views of the NHMRC or its funding partners.

The Norwegian Satellite of the Effective Practice and Organisation of Care (EPOC) Group receives funding from the Norwegian Agency for Development Co-operation (Norad), via the Norwegian Institute of Public Health, to support review authors in the production of their reviews.

We would like to thank the following editors and peer referees who provided comments to improve the protocol: Meghan Bohren, Carol Grech, Margaret Cargo; Marit Johansen for support in developing

the search strategies; and Elizabeth Royle and the Copy Edit Support team for copy-editing the protocol. 


\section{RE F E R E N C E S}

\section{Additional references}

\section{Ames 2017}

Ames HM, Glenton C, Lewin S. Parents' and informal caregivers' views and experiences of communication about routine childhood vaccination: a synthesis of qualitative evidence. Cochrane Database of Systematic Reviews 2017, Issue 2. Art. No: CD011787. [DOI: 10.1002/14651858.CD011787.pub2]

\section{Australian Institute of Health and Welfare 2019}

Australian Institute of Health and Welfare. Admitted patient care 2017-18: Australian hospital statistics. Health services series no. 90. Cat. no. HSE 225. Table S5.26: Separations with hospital-in-the-home care, public and private hospitals, states and territories, 2017-18 2019.

\section{Baxter 2014}

Baxter SK, Blank L, Woods HB, Payne N, Rimmer M, Goyder E. Using logic model methods in systematic review synthesis: describing complex pathways in referral management interventions. BMC Medical Research Methodology 2014;14:62.

\section{Brody 2019}

Brody AA, Arbaje AI, DeCherrie LV, Federman AD, Leff B, Siu AL. Starting up a hospital at home program: facilitators and barriers to implementation. Journal of the American Geriatric Society 2019;67(3):588-95.

\section{Buhagiar 2017}

Buhagiar MA, Naylor JM, Simpson G, Harris IA, Kohler F. Understanding consumer and clinician preferences and decision making for rehabilitation following arthroplasty in the private sector. BMC Health Services Research 2017;17(1):415.

\section{Caplan 2012}

Caplan GA, Sulaiman NS, Mangin DA, Aimonino Ricauda N, Wilson AD, Barclay L. A meta-analysis of "hospital in the home". Medical Journal of Australia 2012;197(9):512-9.

\section{Cochrane EPOC 2017}

Cochrane Effective Practice and Organisation of Care (EPOC). EPOC Qualitative Evidence Syntheses guidance on when to sample and how to develop a purposive sampling frame. EPOC Resources for review authors, 2017. epoc.cochrane.org/epocresources-review-authors.

\section{Critical Appraisal Skills Programme 2020}

Critical Skills Appraisal Programme. CASP (Systematic Review) Checklist. www.casp-uk.net (accessed 7 November 2020).

\section{Deloitte Access Economics 2011}

Deloitte Access Economics. Economic analysis of Hospital in the Home (HiTH). Hospital in the Home Society of Australasia 2011.

\section{Dismore 2019}

Dismore LL, Echevarria C, van Wersch A, Gibson J, Bourke S. What are the positive drivers and potential barriers to implementation of hospital at home selected by low-risk
DECAF score in the UK: a qualitative study embedded within a randomised controlled trial. BMJ Open 2019;9(4):e026609.

\section{Gardner 2003}

Gardner G, Gardner A, Morley G, Watson DA. Managing intravenous medications in the non-hospital setting: an ethnographic investigation. Journal of Infusion Nursing 2003;26(4):227-33.

\section{Gardner 2019}

Gardner M, Shepperd S, Godfrey M, Mäkelä P, Tsiachristas A, Singh-Mehta A, et al. Comprehensive geriatric assessment in hospital and hospital-at-home settings: a mixed-methods study. Health Services and Delivery Research 2019;7(10):1-206.

\section{Glenton 2020}

Glenton C, Bohren MA, Downe S, Paulsen EJ, Lewin S, on behalf of Effective Practice and Organisation of Care (EPOC). EPOC Qualitative Evidence Synthesis: Protocol and review template. Version 1.1. EPOC Resources for review authors. Oslo: Norwegian Institute of Public Health; 2020. Available at: http:// epoc.cochrane.org/epoc-specific-resources-review-authors.

\section{Goncalves-Bradley 2017}

Goncalves-Bradley DC, Iliffe S, Doll HA, Broad J, Gladman J, Langhorne P, et al. Early discharge hospital at home. Cochrane Database of Systematic Reviews 2017, Issue 6. Art. No: CD000356. [DOI: 10.1002/14651858.CD000356.pub4]

\section{Harris 2018}

Harris JL, Booth A, Cargo M, Hannes K, Harden A, Flemming K, et al. Cochrane Qualitative and Implementation Methods Group guidance series-paper 2: methods for question formulation, searching, and protocol development for qualitative evidence synthesis. Journal of Clinical Epidemiology 2018;97:39-48.

\section{HITH Society Australasia 2019}

HITH Society Australasia Ltd. Hospital in the home service register. www.hithsociety.org.au (accessed 10 September 2020).

\section{Hoffmann 2014}

Hoffmann TC, Glasziou PP, Boutron I, Milne R, Perera R, Moher D, et al. Better reporting of interventions: template for intervention description and replication (TIDieR) checklist and guide. BMJ 2014;348:g1687.

\section{Independent Hospital Pricing Authority 2015}

Independent Hospital Pricing Authority (IHPA). Admitted hospital care types: guide for use. www.ihpa.gov.au/ publications/admitted-hospital-care-types (accessed 05 October 2020).

\section{Kraut 2016}

Kraut JC, Singer BJ, Singer KP. Clinician and client views of utilising early supported discharge services. International Journal of Therapy and Rehabilitation 2016;23(10):464-71. 


\section{Lemelin 2007}

Lemelin J, Hogg WE, Dahrouge S, Armstrong CD, Martin CM, Zhang W, et al. Patient, informal caregiver and care provider acceptance of a hospital in the home program in Ontario, Canada. BMC Health Services Research 2007;7:130.

\section{Lewin 2018}

Lewin S, Booth A, Glenton C, Munthe-Kaas H, Rashidian A, Wainwright $\mathrm{M}$, et al. Applying GRADE-CERQual to qualitative evidence synthesis findings: introduction to the series. Implementation Science 2018;13 Suppl 1:2.

\section{Liberati 2009}

Liberati A, Altman DG, Tetzlaff J, Mulrow C, Gotzsche PC, loannidis JP, et al. The PRISMA statement for reporting systematic reviews and meta-analyses of studies that evaluate health care interventions: explanation and elaboration. Annals of Internal Medicine 2009;151(4):W65-94.

\section{Mäkelä 2018}

Mäkelä P, Godfrey M, Cradduck-Bamford A, Ellis G, Shepperd S. A protocol for the process evaluation of a multi-centre randomised trial to compare the effectiveness of geriatricianled admission avoidance hospital at home versus inpatient admission. Trials 2018;19(1):569.

\section{Mäkelä 2020}

Mäkelä P, Stott D, Godfrey M, Ellis G, Schiff R, Shepperd S. The work of older people and their informal caregivers in managing an acute health event in a hospital at home or hospital inpatient setting. Age and Ageing 2020;49(5):856-64.

\section{Marshall 2014}

Marshall MN. Bridging the ivory towers and the swampy lowlands; increasing the impact of health services research on quality improvement. International Journal for Quality in Health Care 2014;26(1):1-5.

\section{NHMRC 2017}

NHMRC Partnership Centre for Health System Sustainability. Advisory groups. www.healthsystemsustainability.com.au/ people-and-partners/advisory-groups/ (accessed 17 February 2020).

\section{NHS Benchmarking Network 2015}

NHS Benchmarking Network. National Audit of Intermediate Care Summary Report 2015. static1.squarespace.com/static/58d8d0ffe4fcb5ad94cde63e/ t/58f08efae3df28353c5563f3/1492160300426/naicreport-2015.pdf (accessed 29 August 2020).

\section{Nowell 2017}

Nowell LS, Norris JM, White DE, Moules NJ. Thematic analysis: striving to meet the trustworthiness criteria. International Journal of Qualitative Methods 2017;16(1):1609406917733847.

\section{Noyes 2020}

Noyes J, Booth A, Cargo M, Flemming K, Harden A, Harris J, et al. Chapter 21: Qualitative evidence. In Higgins JPT, Thomas J, Chandler J, Cumpston M, Li T, Page MJ, Welch VA (editors). Cochrane Handbook for Systematic Reviews of Interventions version 6.1 (updated September 2020), Cochrane, 2020. Available from www.training.cochrane.org/handbook (accessed 05 November 2020).

\section{NVivo [Computer program]}

QSR International Ptd Ltd NVivo. Version 12. Melbourne: QSR International Ptd Ltd, 2020.

\section{OECD 2011}

OECD. Classification of Health Care Functions (ICHA-HC). In: A System of Health Accounts. 2011 edition. Paris: OECD Publishing, 2011.

\section{Shepperd 2016}

Shepperd S, Iliffe S, Doll HA, Clarke MJ, Kalra L, Wilson AD, et al. Admission avoidance hospital at home. Cochrane Database of Systematic Reviews 2016, Issue 9. Art. No: CD007491. [DOI: 10.1002/14651858.CD007491.pub2]

\section{Talley 2007}

Talley RC, Crews JE. Framing the public health of caregiving. American Journal of Public Health 2007;97(2):224-8.

\section{Thomas 2008}

Thomas J, Harden A. Methods for the thematic synthesis of qualitative research in systematic reviews. BMC Medical Research Methodology 2008;8:45.

\section{Tong 2012}

Tong A, Flemming K, Mclnnes E, Oliver S, Craig J. Enhancing transparency in reporting the synthesis of qualitative research: ENTREQ. BMC Medical Research Methodology 2012;12:181.

\section{World Hospital At Home Congress 2019}

World Hospital At Home Congress. Scientific program. whahc2019.kenes.com/2019/programme-(2)/programme.html (accessed 11 August 2020).

\section{Young 2009}

Young J. The development of intermediate care services in England. Archives of Gerontology and Geriatrics 2009;49:S21-5.

\section{AP P E N DICES}

\section{Appendix 1. MEDLINE search strategy}

Ovid MEDLINE(R) and Epub Ahead of Print, In-Process \& Other Non-Indexed Citations, Daily and Versions(R) 1946 to May 6, 2020 


\begin{tabular}{|c|c|c|}
\hline \# & Searches & Results \\
\hline 1 & exp Home Care Services/ & 46956 \\
\hline 2 & exp Hospitalization/ & 236301 \\
\hline 3 & 1 and 2 & 5224 \\
\hline 4 & hospital* at home.ti,ab,kf. & 480 \\
\hline 5 & hospital* in the home.ti,ab,kf. & 206 \\
\hline 6 & (home care and (medical services or health services)).ti,ab,kf. & 708 \\
\hline 7 & $\begin{array}{l}\text { (home based adj (medical care or medical services or health services or } \\
\text { health care or healthcare or care services or care program* or rehab* or thera- } \\
\left.\mathrm{p}^{\star}\right) \text { ).ti,ab,kf. }\end{array}$ & 759 \\
\hline 8 & (home based adj2 hospital based).ti,ab,kf. & 62 \\
\hline 9 & home based program.ti,ab,kf. & 405 \\
\hline 10 & home hospital*.ti,ab,kf. & 456 \\
\hline 11 & home ward.ti,ab,kf. & 42 \\
\hline 12 & rehab* at home.ti,ab,kf. & 152 \\
\hline 13 & 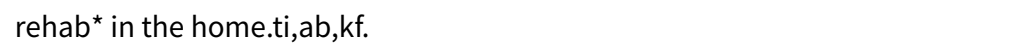 & 68 \\
\hline 14 & rehab* pathway.ti,ab,kf. & 58 \\
\hline 15 & therap* at home.ti,ab,kf. & 373 \\
\hline 16 & therap* in the home.ti,ab,kf. & 184 \\
\hline 17 & admission avoidance.ti,ab,kf. & 125 \\
\hline 18 & avoid* $^{\star}$ admission ${ }^{\star} . t i, a b, k f$. & 205 \\
\hline 19 & (hospital* adj1 avoid $^{\star}$ ).ti,ab,kf. & 1133 \\
\hline 20 & discharge home.ti,ab,kf. & 1304 \\
\hline 21 & home discharge.ti,ab,kf. & 618 \\
\hline 22 & early supported discharge.ti,ab,kf. & 151 \\
\hline 23 & early discharge program*.ti,ab,kf. & 77 \\
\hline 24 & (early discharge adj6 (home or hospital*)).ti,ab,kf. & 749 \\
\hline 25 & or/3-24 & 12379 \\
\hline 26 & Qualitative Research/ & 53846 \\
\hline
\end{tabular}




\begin{tabular}{ll}
27 & Interviews as Topic/ \\
\hline 28 & "Attitude of Health Personnel"/ \\
\hline 29 & $\begin{array}{l}\text { (qualitative or interview* or focus group* or themes or mixed } \\
\text { method?).ti,ab,kf. }\end{array}$ \\
\hline 30 & or/26-29 544030 \\
\hline 31 & 25 and 30 \\
\hline
\end{tabular}

\section{Appendix 2. Data richness scale}

\begin{tabular}{ll}
\hline Score & Measure \\
\hline 1 & $\begin{array}{l}\text { Very little qualitative data presented that relate to the synthesis objective. Those findings that are } \\
\text { presented are fairly descriptive. }\end{array}$ \\
\hline 2 & Some qualitative data presented that relate to the synthesis objective \\
\hline 4 & A reasonable amount of qualitative data that relate to the synthesis objective \\
\hline 5 & A good amount and depth of qualitative data that relate to the synthesis objective \\
\hline
\end{tabular}

Adapted from Ames HMR, Glenton C, Lewin S. Parents' and informal caregivers' views and experiences of communication about routine childhood vaccination: a synthesis of qualitative evidence. Cochrane Database of Systematic Reviews 2017, Issue 2. DOI: 10.1002/14651858.CD011787.pub2

\section{HISTORY}

Protocol first published: Issue 3, 2021

\section{CONTRIBUTIONS OF AUTHORS}

DOC, EG, JW and RB conceived the idea for the review. EG wrote the first draft of the manuscript and DOC, JW, JH, SS, PM, GD, JB and RB contributed to critically revising the drafts for important intellectual content. All authors approved the final version of the manuscript and agree to be accountable for its content.

\section{DECLARATIONS OF INTEREST}

Emma Gearon: none known

Denise O'Connor: Editor with Cochrane EPOC and Cochrane Musculoskeletal Group and director of the Australasian EPOC Satellite. All editorial decisions regarding this work were made independently by another editor.

Jason Wallis: none known

Jia Xi Han: Assistant Managing Editor with EPOC, but has no role in the editorial process of this protocol.

Sasha Shepperd: Co-coordinating editor Cochrane EPOC, but has not been involved in the editorial process of this protocol.

Petra Makela: none known

Rachelle Buchbinder: none known

Review authors involved in the conduct, analysis, and publication of a study that could be included in the review, will not be involved in study eligibility decisions, data extraction, methodological assessment or perform GRADE-CERQual assessments for that study. 


\section{SOURCES OF SUPPORT}

\section{Internal sources}

- Monash Department of Clinical Epidemiology, Cabrini Institute, Australia

Emma Geaorn, Denise O'Connor, Jason Wallis, Jia Xi Han and Rachelle Buchbinder draw a salary

- NSW Health, Australia

Gary Disher draws a salary

- London School of Hygiene and Tropical Medicine, UK

Petra Makela draws a salary

\section{External sources}

- NHMRC Partnership Centre for Health System Sustainability, Australia

This research is conducted by Emma Gearon, Denise O'Connor, Jason Wallis and Rachelle Buchbinder (Cabrini Institute and Monash University) for the NHMRC Partnership Centre for Health System Sustainability (grant ID number: 9100002), administered by the Australian Institute of Health Innovation, Macquarie University. Along with the NHMRC, the funding partners in this research collaboration are: The Bupa Health Foundation; NSW Ministry of Health; Department of Health, WA; and The University of Notre Dame Australia. Their generous support is gratefully acknowledged.

While the NHMRC, The Bupa Health Foundation, NSW Ministry of Health, Department of Health, WA and The University of Notre Dame Australia have provided in-kind and financial support for this research, they have not reviewed the content and are not responsible for any injury, loss or damage however arising from the use of, or reliance on, the information provided herein. The published material is solely the responsibility of the authors and does not reflect the views of the NHMRC or its funding partners. 